
\title{
$\mathrm{UNLV} \mid \underset{\text { LIBRARIES }}{\mathrm{UNIVRSTT}}$
}

\section{Beyond the Dipole Approximation: Angular-Distribution Effects in the 1s Photoemission from Small Molecules}

\author{
Dennis W. Lindle \\ University of Nevada, Las Vegas, lindle@unlv.nevada.edu \\ Oliver Hemmers \\ University of Nevada, Las Vegas, Oliver.Hemmers@unlv.edu
}

H. Wang

University of Nevada, Las Vegas

P. Focke

University of Tennessee, Knoxville

\section{A. Sellin \\ University of Tennessee - Knoxville \\ Follow this and additional works at: https://digitalscholarship.unlv.edu/hrc_fac_articles \\ See next part the for amiditionaleyular and Optical Physics Commons, and the Physical Chemistry Commons}

\section{Repository Citation}

Lindle, D. W., Hemmers, O., Wang, H., Focke, P., Sellin, I. A., Mills, J. D., Sheehy, J. A., Langhoff, P. W. (2000). Beyond the Dipole Approximation: Angular-Distribution Effects in the 1s Photoemission from Small Molecules. AIP Conference Proceedings, 500 156-162.

https://digitalscholarship.unlv.edu/hrc_fac_articles/60

This Conference Proceeding is protected by copyright and/or related rights. It has been brought to you by Digital Scholarship@UNLV with permission from the rights-holder(s). You are free to use this Conference Proceeding in any way that is permitted by the copyright and related rights legislation that applies to your use. For other uses you need to obtain permission from the rights-holder(s) directly, unless additional rights are indicated by a Creative Commons license in the record and/or on the work itself.

This Conference Proceeding has been accepted for inclusion in Environmental Studies Faculty Publications by an authorized administrator of Digital Scholarship@UNLV. For more information, please contact

digitalscholarship@unlv.edu. 
Authors

Dennis W. Lindle, Oliver Hemmers, H. Wang, P. Focke, I. A. Sellin, J. D. Mills, J. A. Sheehy, and P. W. Langhoff 


\section{AIP Conference Proceedings}

\section{Beyond the dipole approximation: Angular distribution effects in the 1s photoemission from small molecules}

D. W. Lindle, O. A. Hemmers, H. Wang, P. Focke, I. A. Sellin et al.

Citation: AIP Conf. Proc. 500, 156 (2000); doi: 10.1063/1.1302648

View online: http://dx.doi.org/10.1063/1.1302648

View Table of Contents: http://proceedings.aip.org/dbt/dbt.jsp?KEY=APCPCS\&Volume=500\&lssue $=1$

Published by the American Institute of Physics.

\section{Related Articles}

High-spin electronic states of lanthanide-arene complexes: $\mathrm{Nd}$ (benzene) and $\mathrm{Nd}$ (naphthalene)

J. Chem. Phys. 136, 204311 (2012)

Comprehensive vacuum ultraviolet photoionization study of the CF3• trifluoromethyl radical using synchrotron radiation

J. Chem. Phys. 136, 204304 (2012)

The electronic spectrum of Si3 I: Triplet D3h system

J. Chem. Phys. 136, 194307 (2012)

Resonant Auger spectroscopy at the carbon and nitrogen K-edges of pyrimidine

J. Chem. Phys. 136, 154308 (2012)

Near-threshold shape resonance in the photoionization of 2-butyne

J. Chem. Phys. 136, 154303 (2012)

\section{Additional information on AIP Conf. Proc.}

Journal Homepage: http://proceedings.aip.org/

Journal Information: http://proceedings.aip.org/about/about_the_proceedings

Top downloads: http://proceedings.aip.org/dbt/most_downloaded.jsp?KEY=APCPCS

Information for Authors: http://proceedings.aip.org/authors/information_for_authors

\section{ADVERTISEMENT}

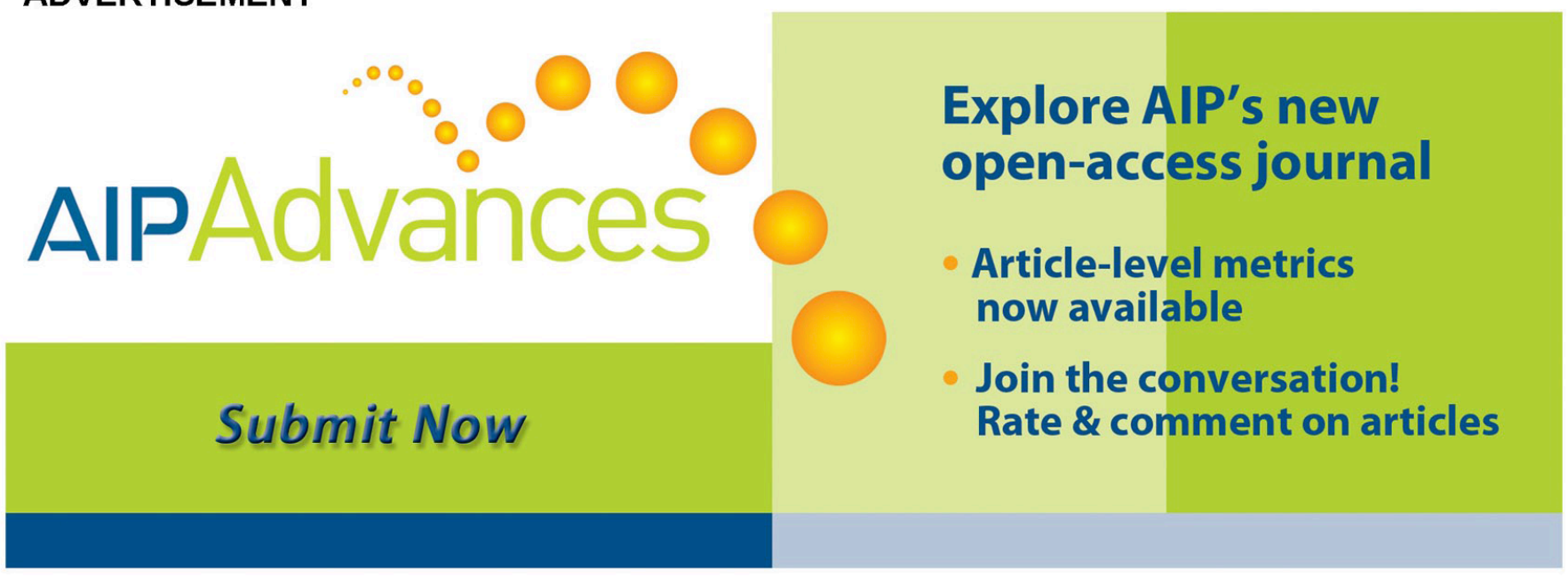




\title{
Beyond the Dipole Approximation: Angular Distribution Effects in the 1s Photoemission from Small Molecules
}

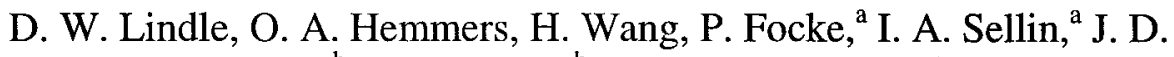 \\ Mills, J. A. Sheehy, ${ }^{b}$ and P. W. Langhoff ${ }^{c}$ \\ Department of Chemistry, University of Nevada, Las Vegas, Nevada, 89154-4003, USA \\ ${ }^{a}$ Department of Physics, University of Tennessee, Knoxville, TN 37996 \\ ${ }^{b}$ Air Force Research Laboratory, AFRL/PRS, Edwards, AFB, CA 93524-7680 \\ ${ }^{c}$ Department of Chemistry, Indiana University, Bloomington, IN 47405
}

\begin{abstract}
Over the past two decades, the dipole approximation has facilitated a basic understanding of the photoionization process in atoms and molecules. Recent experiments on the $1 s$ inner shells of small molecules at relatively low photon energies $(\leq 1000 \mathrm{eV}$ ) show strong nondipole effects. They are significant and measurable at energies close to threshold, in conflict with a common assumption that the dipole approximation is valid for photon energies below $1 \mathrm{keV}$.
\end{abstract}

\section{INTRODUCTION}

The electric-dipole (E1) approximation [1], applied to photoionization, leads to the well-known expression for the differential cross section [2],

$$
\frac{d \sigma}{d \Omega}=\frac{\sigma}{4 \pi}\left[1+\frac{\beta}{2}\left(3 \cos ^{2} \theta-1\right)\right]
$$

which describes the angular distribution of photoelectrons from a randomly oriented sample created by $100 \%$ linearly polarized light. Here, $\sigma$ is the partial photoionization cross section, and $\theta$ is the angle between the vector of the outgoing electron and the vector of linear polarization. The parameter $\beta$ completely describes the angular distribution of photoelectrons, within the dipole approximation. In this approximation, all higher-order interactions, such as electric-quadrupole (E2) and magnetic-dipole (M1), are neglected. This assumption is justified by the argument that the strengths of the $\mathbf{E} 2$ and $\mathbf{M} 1$ interactions relative to electric-dipole effects are approximately equal to the ratio of the photoelectron's velocity to the speed of light [3], a ratio which is small except at very high energies.

CP500, The Physics of Electronic and Atomic Collisions, edited by Y. Itikawa, et al. (C) 2000 American Institute of Physics 1-56396-777-4/00/\$17.00 
Over the past two decades, the dipole approximation has facilitated a basic understanding of the photoionization process in atoms and molecules [2], as well as the application of photoelectron spectroscopy to a wide variety of condensed-phase systems. The first hint of deviations from the dipole approximation was provided by Krause [4] in measurements using unpolarized $x$-rays [5]. A small deviation from the expected dipolar angular distribution at photon energies between 1 and $2 \mathrm{keV}$ was observed and attributed to the influence of $\mathbf{E} 2$ and $\mathbf{M} 1$ interactions. These lowestorder, non-electric-dipole corrections to the dipole approximation lead to so-called nondipole effects in the angular distributions of photoelectrons, described by [6]

$$
\frac{d \sigma}{d \Omega}=\frac{\sigma}{4 \pi}\left[1+\frac{\beta}{2}\left(3 \cos ^{2} \theta-1\right)+\left(\delta+\gamma \cos ^{2} \theta\right) \sin \theta \cos \phi\right]
$$

for $100 \%$ linearly polarized light. The nondipole angular-distribution parameters $\gamma$ and $\delta$ are attributable to interference terms between electric-dipole and electric-quadrupole interactions. Figure 1 describes the geometry and the angles $\theta$ and $\phi$.

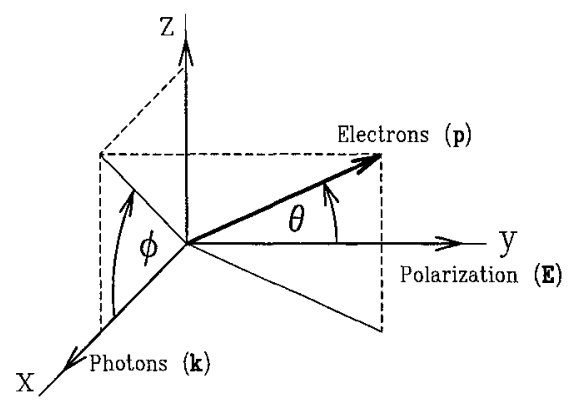

Figure 1. Geometry applicable to photoelectron angular-distribution measurements using polarized light. $\theta$ is the polar angle between the photon polarization vector $\varepsilon$ and the momentum vector $\mathbf{p}$ of the photoelectron. $\phi$ is the azimuthal angle defined by the photon propagation vector $\mathbf{k}$ and the projection of $p$ into the $x-z$ plane.

More-recent measurements [7,8], focussing on noble-gas core levels (Ar $K$ and $\mathrm{Kr}$ $L$ ) and photon energies above $2 \mathrm{keV}$, have begun to investigate nondipole effects in photoelectron angular distributions in more detail. In contrast, the present experiment concentrates on the $\mathrm{N}_{2} \mathrm{~N} 1 s$ and CO C1s inner shells at relatively low photon energies (300 to $700 \mathrm{eV}$ ). Nondipole effects are observed to be large and highly energy dependent in this region, especially close to core-level thresholds, in conflict with a common assumption in applications of photoelectron spectroscopy; namely, that the dipole approximation is valid for photon energies below $1 \mathrm{keV}$. The potential significance of these findings is nicely illustrated by comparison of the present results for the $\mathrm{N}_{2}$ and the $\mathrm{CO} \gamma_{1 s}$ parameters with theories for atomic nitrogen and atomic carbon [9], where the influence of nondipole effects are expected to be much smaller. 


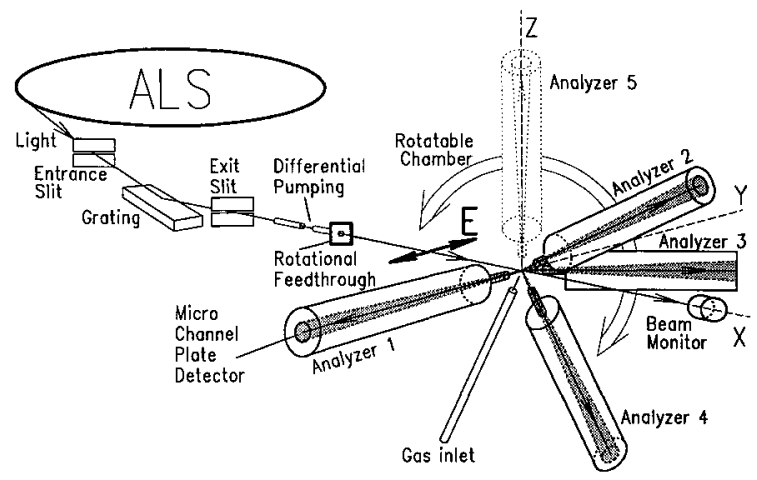

Figure 2. Experimental schematic of the electron time-of-flight system. Light from the ALS storage ring passes through beamline optics into a differential-pumping section. The chamber and analyzers can rotate around the photon beam for more accurate electron angular-distribution measurements.

\section{EXPERIMENT}

The experiments were performed on undulator beamline 8.0, [10], which covers the $100-1500 \mathrm{eV}$ photon-energy range. The monochromator entrance slit was set to $70 \mu \mathrm{m}$ and the exit slit to $100 \mu \mathrm{m}$ yielding very high flux, because high photon resolution was not needed. During the measurements the ALS operated at $1.9 \mathrm{GeV}$ in two-bunch mode with a photon pulse every 328 ns. Four time-of-flight (TOF) electron analyzers, equipped with microchannel plates for electron detection, collect spectra simultaneously at different angles. The total electron flight paths are $437.5 \mathrm{~mm}$, and the analyzers have a full cone acceptance angle of $5.4^{\circ}$.

The interaction region is formed by an effusive gas jet intersecting the photon beam which has a diameter of less than $1 \mathrm{~mm}$. Energy resolution of the TOF analyzers with a focus size of $1 \mathrm{~mm}$ is $1 \%$ of the electron kinetic energy. Each spectrum was collected for about $600 \mathrm{~s}$. The gas samples were obtained either commercially (CO) or directly from ambient air $\left(\mathrm{N}_{2}\right)$. A mixture of the sample with xenon was used sometimes because Xe has an abundance of Auger lines below $100 \mathrm{eV}$ kinetic energy, which provide excellent internal calibration for each spectrum.

\section{RESULTS}

Figure 3 shows two superimposed spectra, both taken at the magic angle $\theta=54.7^{\circ}$, but at different $\phi$ angles. The spectra were measured close to the C1s threshold (296 $\mathrm{eV}$ ) and are scaled to the area of the Xe NOO Auger lines and the obvious intensity differences between the $\mathrm{CO} \mathrm{C} 1 s$ peaks in the two spectra are due entirely to nondipole 


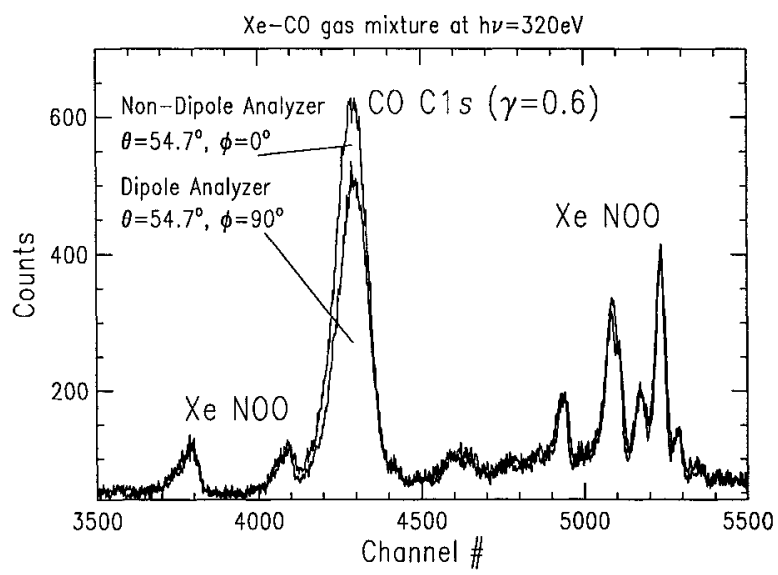

Figure 3. Photoelectron spectra of a CO-Xe mixture measured at a photon energy of $320 \mathrm{eV}$. One spectrum was taken with the dipole magic-angle analyzer and the other spectrum with the nondipole analyzer. The spectra are normalized to the Xe NOO Auger lines. The intensity differences in the CO $\mathrm{C} 1 s$ lines between the two analyzers is due entirely to nondipole effects.

effects because both spectra are at the magic angle where the $\beta$ parameter has no influence.

For the dipole magic-angle analyzer the differential cross section in Eq. (2) reduces to the partial cross section; E2 and M1 effects vanish in the $\phi=90^{\circ}$ plane even if relativistic effects are included [11]. For the nondipole analyzer,

$$
\frac{d \sigma}{d \Omega}=\frac{\sigma}{4 \pi}\left[1+\sqrt{\frac{2}{27}}(3 \delta+\gamma)\right]=\frac{\sigma}{4 \pi}\left[1+\sqrt{\frac{2}{27}} \zeta\right]
$$

which simplifies further for $s$ subshells $[6,12]$ in the non-relativistic approach where $\delta$ vanishes. We are using $\zeta=3 \delta+\gamma$ for measurements that don't resolve the $\delta$ and $\gamma$ parameters of the angular distributions. In the case of molecular effects it is not clear if the $\delta$ parameter for $s$-shells vanishes near threshold.

With our experimental geometry, it is possible to measure the $\zeta$ parameter for $s$ subshells directly, if the degree of linear polarization is known, by using the two magic angle analyzers. The data points for $\mathrm{CO}$ and $\mathrm{N}_{2}$ in Figures 4 and 5 show strong nondipole contributions with maxima of $\zeta=1.2$.

The difference between $\mathrm{CO}$ and $\mathrm{N}_{2}$ lies in the position of the maxima. For $\mathrm{N}_{2}$ the maximum is about $60 \mathrm{eV}$ above the $\mathrm{N}_{2} 1 s$ ionization threshold much higher than the maximum of the dipole shape resonance at about $420 \mathrm{eV}$. The maximum of the $\zeta$ parameter for the $\mathrm{CO} \mathrm{C} 1 s$ is close to the maximum of the dipole shape resonance at $305 \mathrm{eV}$. 


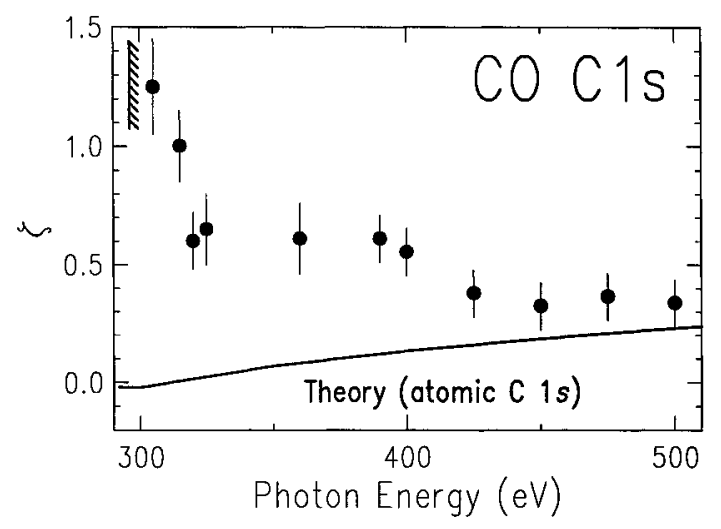

Figure 4. Electron angular anisotropy parameter $\zeta$ for the $\mathrm{CO} \mathrm{Cl} s$ photoline from threshold to $\mathrm{hv}=500$ $\mathrm{eV}$. The theoretical curve for atomic carbon is from Lajohn and Pratt [9].

A qualitative explanation for the behavior of $\zeta$ can be obtained from the following model. Just as molecular $\beta$ values can change rapidly with photon energy for large differences in polarization components for ionization along and perpendicular to a molecular axis (due to a resonance, for example), so also $\zeta$ values can behave similarly but with greater sensitivity to the difference in polarization components because of the higher power of the transition moment coordinate involved. Thus, the observed molecular $\zeta$ effects may be universal.

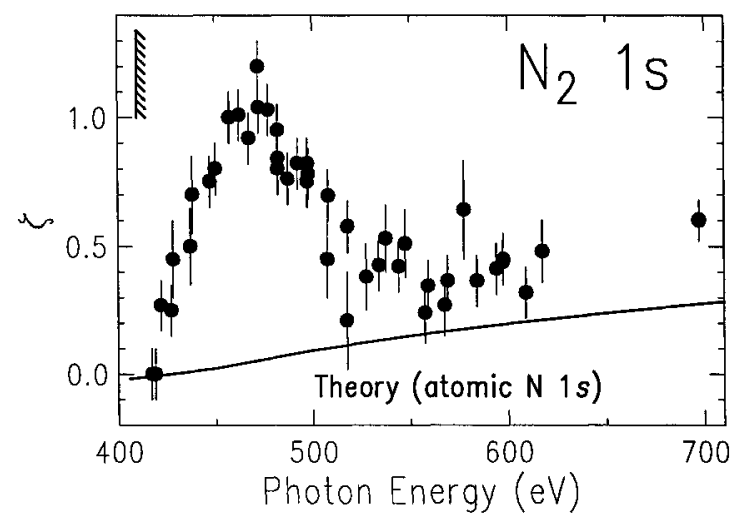

Figure 5. Electron angular anisotropy parameter $\zeta$ for the $\mathrm{N}_{2} 1 s$ photoline from threshold to $h v=700$ $\mathrm{eV}$. The theoretical curve for atomic nitrogen is from Lajohn and Pratt [9]. 


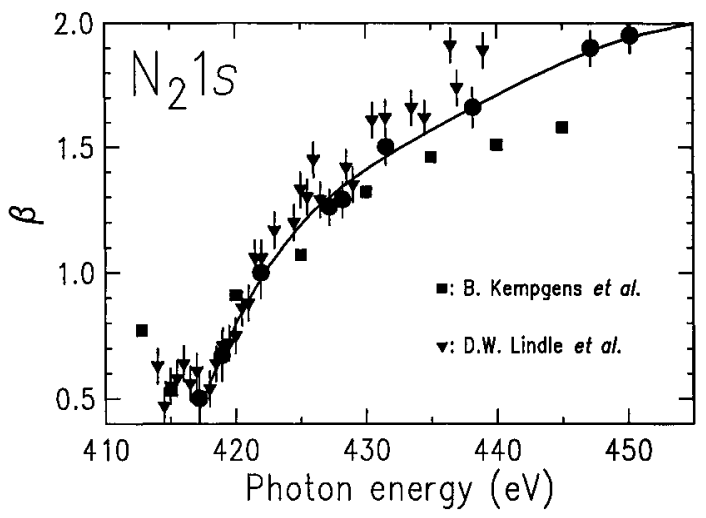

Figure 6. Electron angular anisotropy parameter $\beta$ for the $\mathrm{N}_{2} 1 s$ photoline from threshold to $h v=450$ $\mathrm{eV}$. Measurements by Kempgens [14] and Lindle [15] did not take non-dipolar effects into account and deviate from our measurements.

The variations of $\beta$ (and $\zeta$ ) with photon energy in atoms are due to the interference of different partial waves ( $p \rightarrow s$ and $d$, for example) whereas in molecules this can be due to the interference of the polarization components $\left(1 \mathrm{~s} \rightarrow p \sigma\right.$ and $p \pi$ in $\mathrm{N}_{2}$, for example).

One consequence of these strong molecular nondipole effects near threshold is the possibility of influences on previous measurements of $\beta$-parameters, as demonstrated in Fig. 6 . If the $\beta$-parameter is not measured in the plane perpendicular to the direction of the light (and linear polarized light) the intensities used to determine $\beta$ are influenced by the forward/backward intensities of $\zeta$. These intensities reduce or increase the $\beta$ values as shown in Fig. 6 for Kempgens and Lindle. Larger values of $\zeta$ lead to larger deviations in $\beta$.

The present results illustrate that any photoemission experiment, whether on gases, solids, or surfaces, can be influenced by nondipole effects at relatively low photon energies, pointing to a general need for caution in interpreting angle-resolved photoemission data.

\section{ACKNOWLEDGMENTS}

The authors thank the staff of the ALS and the IBM, LBNL, LLNL, the University of Tennessee, and Tulane University collaboration for their support. This research is funded by the NSF (PHY-9303915), the DOE Nevada EPSCoR. The ALS is supported by the U.S. DOE through the Materials Science Division, Office of Basic Energy Sciences, Office of Energy Research at the Lawrence Berkeley National Laboratory under contract No. DE-AC03-76SF00098. 


\section{REFERENCES}

1. Bethe, H. A. and Salpeter, E. E., Quantum Mechanics of One- and Two-Electron Atoms, Berlin: Springer-Verlag, 1957.

2. Manson, S. T., and Dill, D., Electron Spectroscopy: Theory, Techniques, and Applications, New York: Academic, 1978, Vol. 2, edited by Brundle, C. R. and Baker, A. D. (Academic, New York, 1978).

3. Cooper, J. and Zare, R. N., J. Chem. Phys. 48, 942 (1968).

4. Krause, M. O., Phys. Rev. 177, 151 (1969).

5. For unpolarized incident light, $\beta / 2$ is replaced by $\beta / 4$ in Eq. (1), and $\theta$ is measured between the propagation vectors of the photon and the photoelectron. Otherwise, the essential physics is the same.

6. Cooper, J. W., Phys. Rev. A 42, 6942 (1990); 45, 3362 (1992); 47, 1841 (1993).

7. Krässig, B., Jung, M., Gemmell, D. S., Kanter, E. P., LeBrun, T., Southworth, S. H., and Young, L., Phys. Rev. Lett. 75, 4736 (1995).

8. Jung, M., Krässig, B., Gemmell, D. S., Kanter, E. P., LeBrun, T., Southworth, S. H., and Young, L., Phys. Rev. A 54, 2127 (1996).

9. Lajohn, L. and Pratt, R. H., (private communication)

10. Perera, R. C. C., Nucl. Instrum. Methods A319, 277 (1992).

11. Scofield, J. H., Phys. Rev. A 40, 3054 (1989); Phys. Scripta 41, 59 (1990).

12. Amusia, M. Ya., Arifov, P. U., Baltenkov, A. S., Grinberg, A. A., and Shapiro, S. G., Phys. Lett. 47A, 66 (1974); Amusia, M. Ya., Baltenkov, A. S., Grinberg, A. A., and Shapiro, S. G., Sov. Phys.-JETP 41, 14 (1975); Amusia, M. Ya. and Cherepkov, N. A., Case Studies in Atomic Physics, Amsterdam: North-Holland, 1975, Vol. 5.

13. Hemmers, O., Whitfield, S. B., Glans, P., Wang, H., Lindle, D. W., Wehlitz, R., and Sellin, I. A., Rev. Sci. Instrum. 69, 3809 (1998).

14. Kempgens, B., Kivimäki, A., Neeb, M., Köppe, H.M., Bradshaw, A.M., and Feldhaus, J., J. Phys. B 29 (1996).

15. Lindle, D. W., Truesdale, C.M., Kobrin, P.H., Ferrett, T.A., Heimann, P.A., Becker, U., Kerkhoff, H.G., and Shirley, D.A., J. Chem. Phys. 81 (1984). 